\title{
An Arc Fault Detection Method Based on Multidictionary Learning
}

\author{
Na Qu (D), ${ }^{1,2}$ Jianhui Wang, ${ }^{2}$ Jinhai Liu, $^{2}$ and Zhi Wang' \\ ${ }^{1}$ Liaoning Key Laboratory of Aircraft Safety and Airworthiness, Shenyang Aerospace University, Shenyang, Liaoning, China \\ ${ }^{2}$ School of Information Science and Engineering, Northeastern University, Shenyang, Liaoning, China \\ Correspondence should be addressed to $\mathrm{Na} \mathrm{Qu;11502332@qq.com}$
}

Received 31 July 2018; Revised 23 October 2018; Accepted 19 November 2018; Published 3 December 2018

Academic Editor: Rafal Zdunek

Copyright (c) $2018 \mathrm{Na}$ Qu et al. This is an open access article distributed under the Creative Commons Attribution License, which permits unrestricted use, distribution, and reproduction in any medium, provided the original work is properly cited.

\begin{abstract}
This paper uses the dictionary learning of sparse representation algorithm to detect the arc fault. Six kinds of characteristics, that is, the normalized amplitudes of $0 \mathrm{~Hz}, 50 \mathrm{~Hz}, 100 \mathrm{~Hz}, 150 \mathrm{~Hz}, 200 \mathrm{~Hz}$, and $250 \mathrm{~Hz}$ in the current amplitude spectrum, are used as inputs. The output is normal work or arc fault. Increasing the number of training samples can improve the accuracy of the tests. But if the training samples are too many, it is difficult to be expressed by single dictionary. This paper designs a multidictionary learning method to solve the problem. Firstly, $n$ training samples are selected to form $s$ overcomplete dictionaries. Then a dictionary library consisting of $s$ dictionaries is constructed. Secondly, $t(t \leq s)$ dictionaries are randomly selected from the dictionary library to judge the test results, respectively. Finally, the final detest result is obtained through the maximum number of votes, that is, the modality with the most votes is the detest result. Simulation results show that the accuracy of detection can be improved.
\end{abstract}

\section{Introduction}

Electrical fire generally refers to high temperature, arc, and spark ignition because of the fault of electrical wiring, electrical equipment, and power supply equipment. Statistical analysis shows that the proportion of electric fires caused by arc fault is up to $50 \%$. Therefore, the detection of arc fault is meaningful to ensure electrical safety.

UL1699 standard for arc fault protection is proposed in 1999. A new apparatus named Arc Fault Circuit Interrupter (AFCI) is introduced. AFCI is a circuit breaker to detect arc fault and cut off the circuit so as to reduce the damage. In recent years, many scholars have begun to study the arc fault. Generally, current is selected as the detection signal of arc fault. Most of the researches on arc fault current detection are based on the zero rest. The wavelet transform method is always used to detect arc fault [1-8]. In addition to wavelet transform, a few other methods have been studied to detect the arc fault. The ABCD method is to establish the relationship between input and output current through a transfer matrix. The coefficients of ABCD matrix depend on conductor parameters, arc fault, and load types. It is mainly applied to aircraft arc fault detection [9]. The arc fault current detection algorithm based on the theory of quantum probability model is mainly applied to the photovoltaic power system [10]. The method of Chirp-zeta transform is used to analyze the low frequency harmonic of the current signal [11].

Sparse representation classification has recently attracted the interests of many researchers and has been successfully applied to several problems such as robust face recognition, visual tracking, and transient acoustic signal classification. In [12], a tracking model of bidirectional sparse representation is established. The model achieves uniform convergence through $L_{2}$ norm constraint and the sparse correlation coefficient matrix. In [13], the models and the algorithms of comprehensive dictionary, analytic dictionary, and blind dictionary are introduced. The typical applications of dictionary learning methods are illustrated. In [14], a new SAR target recognition method, which is based on the discriminative dictionary learning and joint dynamic sparse representation model, is proposed. In [15], the method of nonnegative matrix decomposition (NMF) concretely embodies the concept of sparse representation and provides a complete Matlab code and extension method. In [16], a 


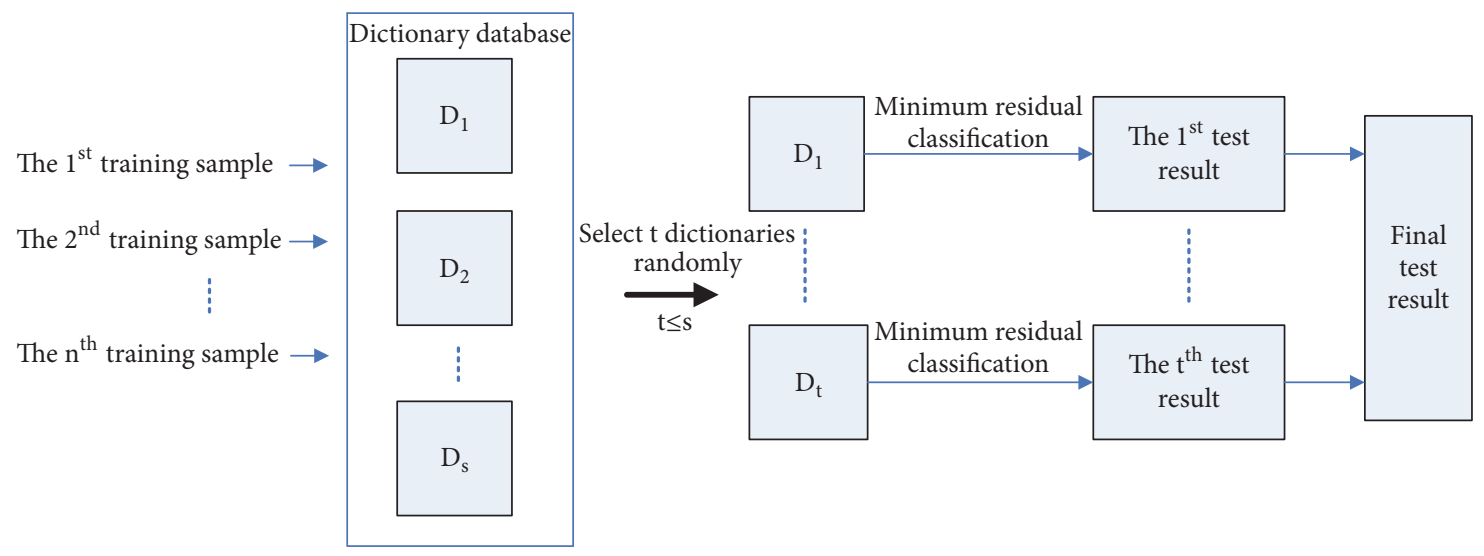

FIGURE 1: Multidictionary learning scheme.

discriminative dictionary, which is learned by K-SVD and LC-KSVD algorithm, is presented. In [17], two dictionary learning methods based on Fisher discrimination dictionary learning (FDDL) are proposed. In [18], a multimodal taskdriven dictionary learning algorithm under the joint sparsity constraint (prior) to enforce collaborations among multiple homogeneous/heterogeneous sources of information is proposed. In [19], a sparse representation model based on joint kernel is proposed to complete key-frame extraction for human motion capture data. In [20], the problem of transmit beam pattern matching design for multiple-input multiple-output (MIMO) radar is addressed within a sparse representation framework.

In this paper, the multidictionary learning method of sparse representation is studied and applied to arc fault detection to improve the accuracy.

\section{Multidictionary Learning Method}

Sufficient training samples of the $i^{\text {th }}$ modality are given [21]. The test sample $y$ from the same modality will approximately lie in the linear span of the training samples associated with $i^{\text {th }}$ object. We define a new matrix $D=\left[\begin{array}{lll}D^{1} & D^{2} D^{3} \ldots\end{array}\right.$ $D^{n}$ ] for the entire training set as the concatenation of all $n$ object modalities. Then, the linear representation of $y$ can be rewritten in terms of all training samples as follows:

$$
y=D x_{0}
$$

$\boldsymbol{x}_{0}=\left[0, \cdots, 0, \alpha_{1} \cdots \alpha_{m}, \cdots 0, \cdots 0\right]^{T}$ is a coefficient vector whose entries are zero except those associated with the $i^{\text {th }}$ modality. As the entries of the vector $x_{0}$ encode the identity of the test sample $y$, it is tempting to attempt to obtain it by solving the linear system of equation $\boldsymbol{D} \boldsymbol{x}=\boldsymbol{y}$. There may be an infinite number of solutions to the underdetermined linear equations. In order to reduce the range of selection to a satisfactory solution, it is necessary to increase condition. A function $\boldsymbol{J}(\boldsymbol{x})$ is introduced to rationally evaluate the candidate solution of $\boldsymbol{x}$, and its value is expected to be as small as possible [22], usually in the form of $L_{p}$ norm, as follows:

$$
\begin{aligned}
\widehat{\boldsymbol{x}}=\underset{x}{\arg \min } & \|\boldsymbol{x}\|_{p} \\
\text { s.t. } & \boldsymbol{D} \boldsymbol{x}=\boldsymbol{y} .
\end{aligned}
$$

When $p \leq 1$, sparse solution $\widehat{\boldsymbol{x}}$ can be obtained. Then, a sparse representation model is formed, and $\boldsymbol{D}$ is the learning dictionary. In practical applications, formula (2) is usually written in the form of formula (3) due to the existence of errors.

$$
\begin{aligned}
\widehat{\boldsymbol{x}}=\underset{x}{\arg \min } & \|\boldsymbol{x}\|_{p} \\
\text { s.t. } & \|\boldsymbol{y}-\boldsymbol{D} \boldsymbol{x}\|_{2}^{2} \leq \varepsilon
\end{aligned}
$$

$\varepsilon$ is the error threshold; $\boldsymbol{y}=\left[y_{1}, \cdots, y_{n}\right]^{T}$ is a test vector. The overcomplete dictionary is composed of training samples. Increasing the number of training samples can improve the accuracy of the tests. But if the number of training samples is too much, it is difficult to be expressed by single dictionary. This paper designs a multidictionary learning method to solve the problem. The multidictionary learning scheme is shown in Figure 1. Firstly, $n$ training samples are selected to form $s$ overcomplete dictionaries. Then a dictionary library consisting of $s$ dictionaries is constructed. Secondly, $t(t \leq s)$ dictionaries are randomly selected from the dictionary library to judge the test results, respectively. Finally, the final detest result is obtained through the maximum number of votes; that is, the modality with the most votes is the detest result.

The minimum residual classification is used to determine the detest result of single-dictionary learning. $\widehat{x}$ is the projection coefficient of the test sample $y$ on the dictionary $\boldsymbol{D}$ of the $i^{\text {th }}$ modality. When $y$ belongs to the $i^{\text {th }}$ modality, $\widehat{\boldsymbol{y}}_{i}=\boldsymbol{D} \widehat{\boldsymbol{x}}$ approximates $\boldsymbol{y}$. The smaller distance between $\widehat{y}_{\boldsymbol{i}}$ and $\boldsymbol{y}$ (i.e., residual) is, the greater possibility that $\hat{y}_{\boldsymbol{i}}$ belongs to $i^{\text {th }}$ modality is, as shown in the following formula:

$$
\boldsymbol{r}_{i}(\boldsymbol{y})=\min _{i}\left(\|\boldsymbol{y}-\boldsymbol{D} \hat{\boldsymbol{x}}\|_{2}\right)
$$



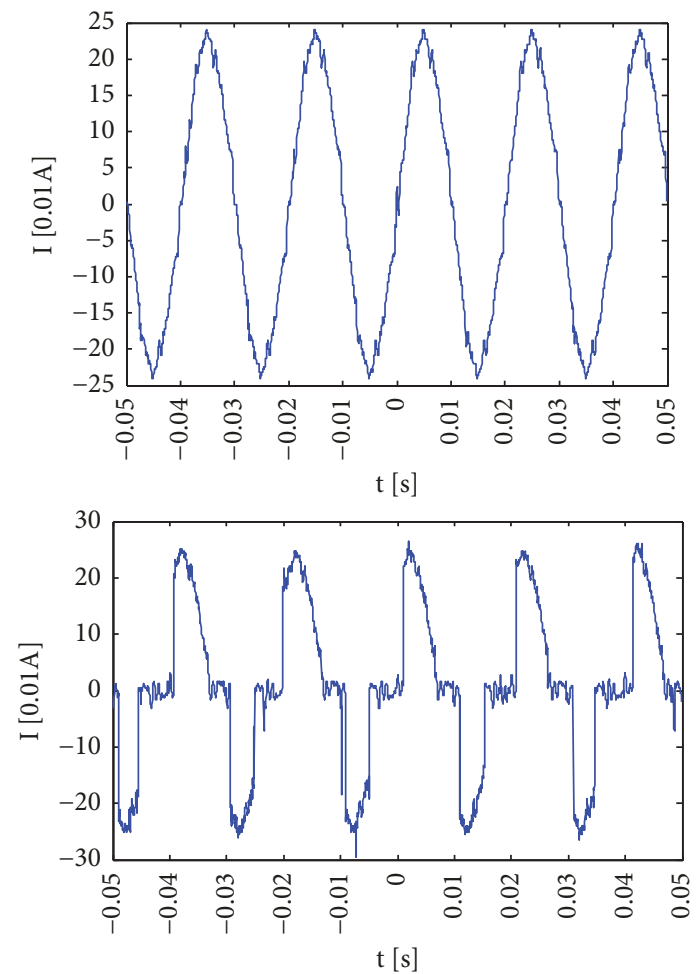

FIGURE 2: Current waveform of resistive load: (a) normal work; (b) arc fault.

\section{Sample Data Acquisition}

Sample data are obtained by an arc fault experimental platform. The experimental platform consists of power supply, arc fault generator, data acquisition device, loads, switch, and so on [23]. Power supply is " $220 \mathrm{~V}, 50 \mathrm{~Hz}$ " AC power. Arc fault generator is mainly composed of fixing carbon electrode and moving copper electrode. The data acquisition device consists of a sampling resistance and an oscilloscope. TDS1001C-SC of Tektronix oscilloscope and TPP0101 10X of voltage probe are selected. Sample interval is $4 \times 10^{-4} \mathrm{~s}$. The current waveform of the circuit is obtained by the sampling resistance method. The selection of sampling resistance is different depending on the load. The loads that are commonly used in family and woke, such as resistive load, resistive and inductive load, DC motor load, series motor load, eddy current load, and switching power load, are chosen. " $220 \mathrm{~V}, 100 \mathrm{~W}$ " incandescent lamp is selected as resistive load. " $220 \mathrm{~V}, 100 \mathrm{~W}$ " incandescent lamp and $22 \mathrm{mH}$ inductors are selected as resistive and inductive load. The DC motor load selects the high grade position of electric blower. The series motor load selects an electric drill. The eddy current load selects the induction cooker. The switch power load selects the computer. The current data of normal work and arc fault are collected under the different types of loads conditions. The current waveform is shown in Figures 2-7. The sampling resistance is $100 \mathrm{ohms}$ when the loads are resistive load and resistive and inductive load. The unit of ordinate is $0.01 \mathrm{~A}$ in Figures 2 and 3. The sampling resistance is $50 \mathrm{ohms}$ when the loads are DC motor load, series motor load, and switching power load. The unit
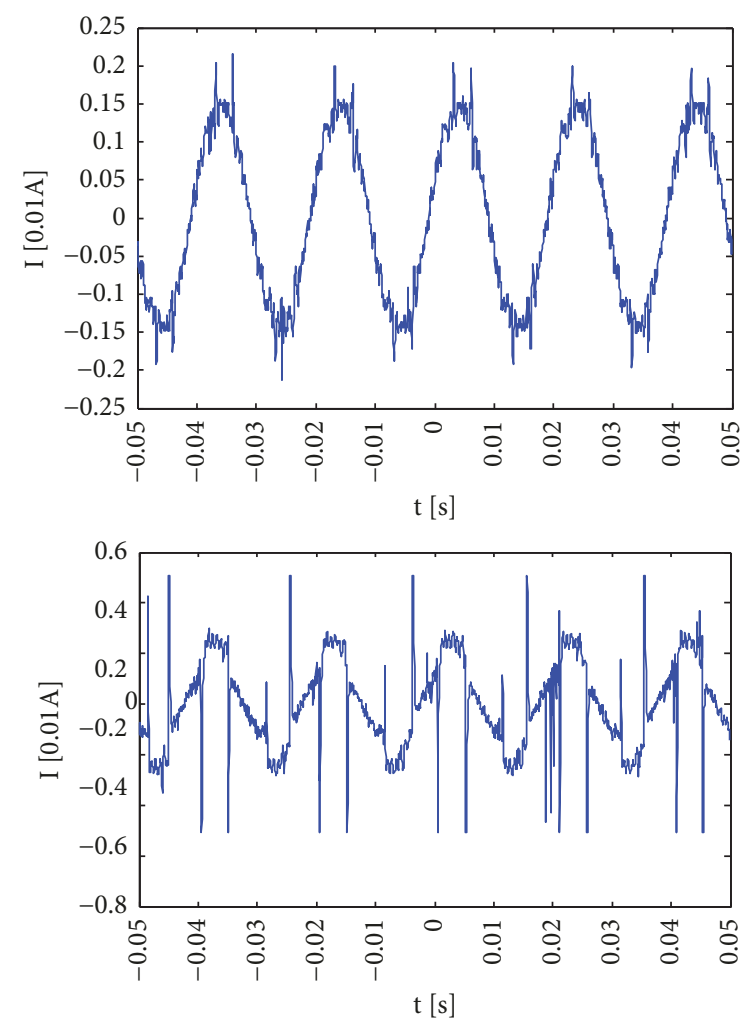

FIgURE 3: Current waveform of resistive and inductive load: (a) normal work; (b) arc fault.

of ordinate is $0.02 \mathrm{~A}$ in Figures 4, 5, and 7. The sampling resistance is $1 \mathrm{ohm}$ when the load is eddy current load. The unit of ordinate is $1 \mathrm{~A}$ in Figure 6.

In the current waveform of arc fault, there is zero rest (i.e., nearly flat zero current segments around the normal zero crossing of the waveform) under the resistive load, the DC motor load, and series motor load conditions. When the load is the computer, the normal current waveform also appears to be zero rest. The zero rest of current waveform is not obvious when the arc fault occurs under the resistive and inductive load condition. Therefore, the arc fault cannot be detected accurately by the zero rest.

The current amplitude spectrum is obtained by Fourier transform. The current signal is decomposed into the Fourier series of trigonometric functions, which is shown in the following formula:

$$
f(t)=a_{0}+\sum_{n=1}^{\infty}\left[a_{n} \cos \left(n \omega_{1} t\right)+b_{n} \sin \left(n \omega_{1} t\right)\right]
$$

In formula (5), $a_{0}=(1 / T) \int_{t_{0}}^{t_{0}+T_{1}} f(t) d t, a_{n}=$ $\left.T_{1}\right) \int_{t_{0}}^{t_{0}+T_{1}} f(t) \cos \left(n \omega_{1} t\right) d t$, and $b_{n}=\left(2 / T_{1}\right) \int_{t_{0}}^{t_{0}+T_{1}} f(t) \sin \left(n \omega_{1} t\right) d t$, $n=1,2,3, \cdots$.

Formula (6) can be obtained by formula (5).

$$
f(t)=d_{0}+\sum_{n=1}^{\infty}\left[d_{n} \sin \left(n \omega_{1} t+\theta_{n}\right)\right]
$$



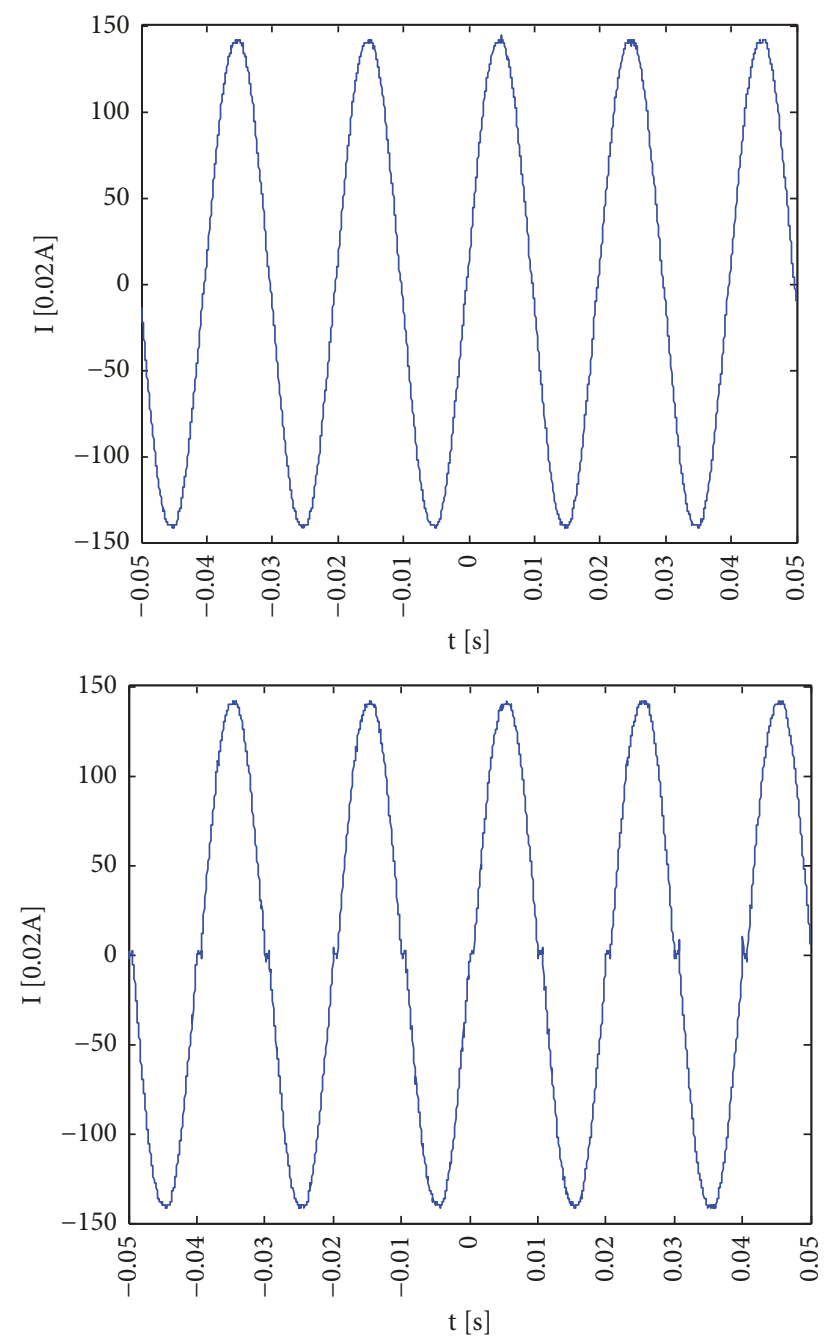

FIGURE 4: Current waveform of DC motor load: (a) normal work; (b) arc fault.

In formula (5), $d_{0}=a_{0}, d_{n}=\sqrt{a_{n}{ }^{2}+b_{n}{ }^{2}}$, and $\theta_{n}=$ $\arctan \left(a_{n} / b_{n}\right)$. The $\mathrm{x}$-coordinate of the amplitude spectrum is made up of $n \omega_{1}$. The $y$-coordinate of the amplitude spectrum is made up of $d_{n}$.

One set of current amplitude spectrums and standardized values is shown in the appendix for Table 1. Compared with the spectrum of normal work, that of arc fault has different distribution characteristics.

\section{Arc Fault Detection}

4.1. Single-Dictionary Learning. The dictionary matrix consists of $n$ modalities of training samples, that is, $D=\left[D^{1} D^{2}\right.$ $D^{3} \ldots D^{n}$. The number of rows in the matrix $D$ is the number of sample characteristics [23]. In this paper, six kinds of characteristics, that is, the normalized amplitudes of $0 \mathrm{~Hz}, 50 \mathrm{~Hz}, 100 \mathrm{~Hz}, 150 \mathrm{~Hz}, 200 \mathrm{~Hz}$, and $250 \mathrm{~Hz}$ in the current amplitude spectrum, are used. The values are between 0 and 1. The two modalities of training samples are normal work and arc fault; that is, $n=2$. The number of columns is the total
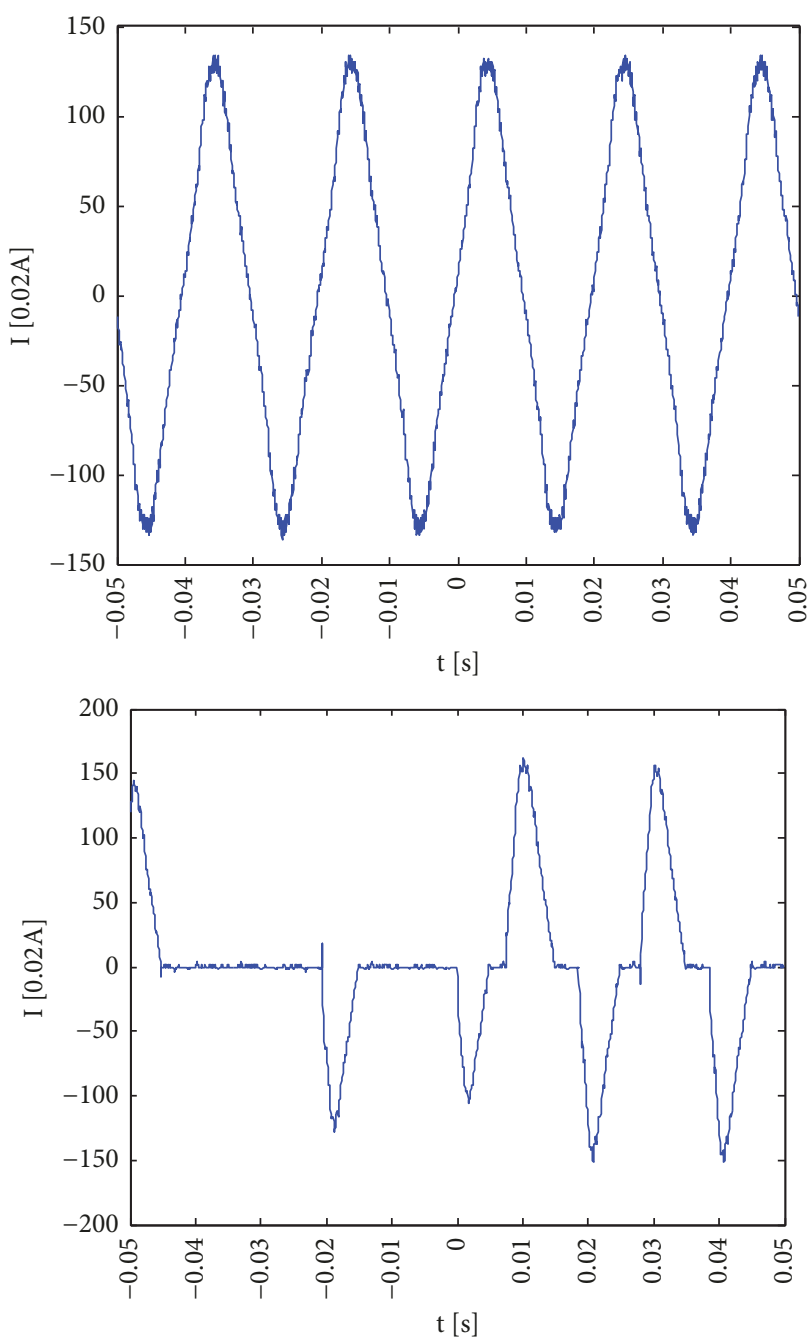

FIGURE 5: Current waveform of series motor load: (a) normal work; (b) arc fault.

number of two modalities of training samples; that is, $D=\left[D^{1}\right.$ $D^{2}$.

4.2. Multidictionary Selection. Ten sets of dictionaries are selected to form the dictionary library. In order to give consideration to accuracy and instantaneity, five sets of dictionaries are selected randomly from the dictionary library. $L_{3 / 4}$ norm regularization algorithm with good sparsity and accuracy is selected to identify the classification [24], as shown in the following formula:

$$
\begin{aligned}
\hat{\boldsymbol{x}}_{L 3 / 4}=\underset{x}{\arg \min } & \|\boldsymbol{x}\|_{3 / 4} \\
\text { s.t. } & \|\boldsymbol{y}-\boldsymbol{D} \boldsymbol{x}\|_{2}^{2} \leq \boldsymbol{\varepsilon}
\end{aligned}
$$

Formula (8) can be obtained by formula (7).

$$
\widehat{\boldsymbol{x}}_{L 3 / 4}=\underset{x}{\arg \min }\left\{\frac{1}{n} \sum_{i=1}^{n}\left(y_{\boldsymbol{i}}-\boldsymbol{D}_{\boldsymbol{i}} \boldsymbol{x}\right)^{2}+\lambda \sum_{i=1}^{p}\left|\boldsymbol{x}_{i}\right|^{3 / 4}\right\}
$$



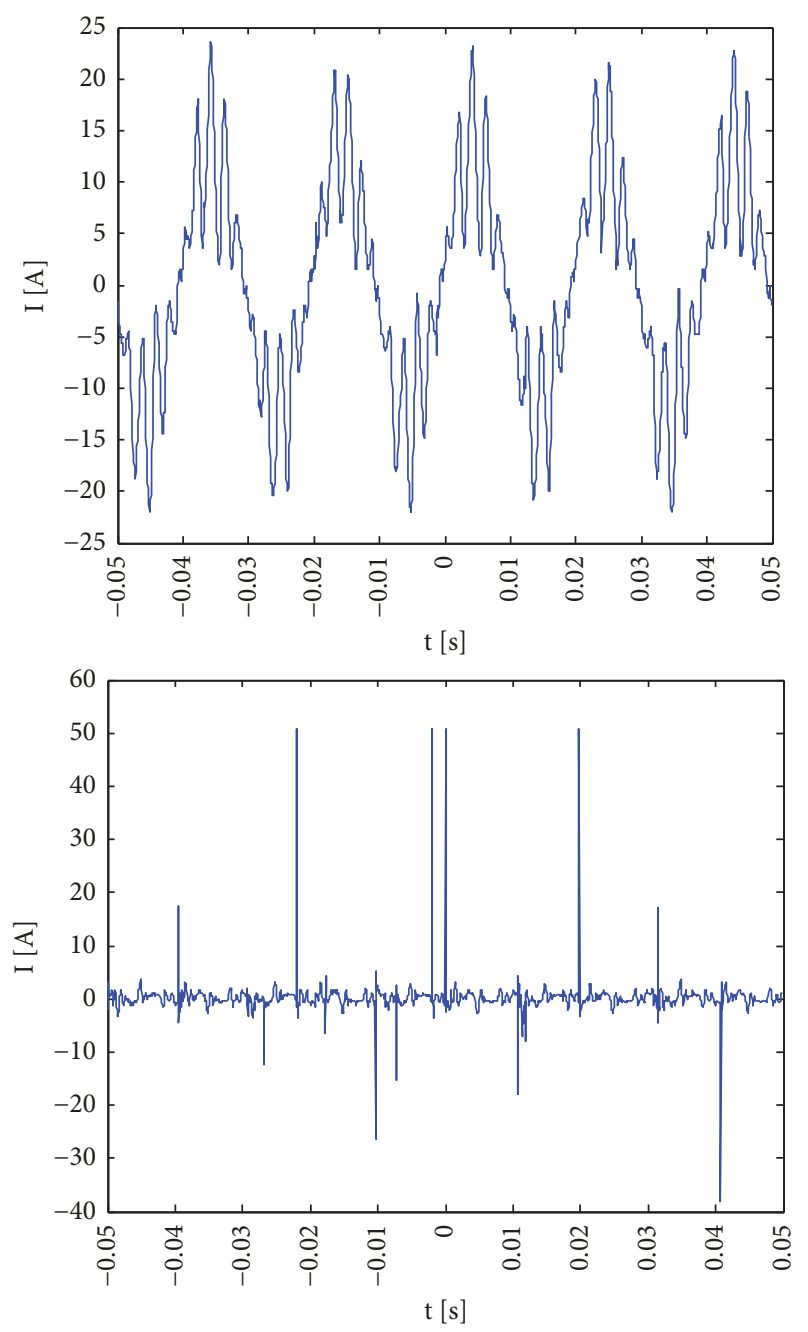

FIGURE 6: Current waveform of eddy current load: (a) normal work; (b) arc fault.

$\boldsymbol{y}_{i}$ represents the $i^{\text {th }}$ modality test sample; $\lambda$ is the regularization parameter. $P$ is the number of samples of each modality. In the later application, $P=18 . \boldsymbol{x}_{i}$ is the $i^{\text {th }}$ training sample of each modality.

4.3. Test and Simulation. Five sets of dictionaries are selected randomly from the dictionary library, that is, $D_{1}, D_{2}, D_{3}, D_{4}$, and $\boldsymbol{D}_{5}$. The test sample comes from the arc fault spectrum of resistive and inductive load; $y=[0.02 ; 1 ; 0.016 ; 0.27 ; 0.081 ; 0.158]$. The detection result is shown in Figure 8 when the single dictionary is $\boldsymbol{D}_{\boldsymbol{1}}$. In Figure 8(a), the horizontal axis 1-18 is the training samples number of normal work. The horizontal axis $19-36$ is the training samples number of the arc fault. The training samples come from current amplitude spectrum under the condition of resistive load, resistive and inductive load, DC motor load, series motor load, eddy current load, and switching power load. The fault detection result is determined by the residual comparison and projected coefficients comparison of two modalities. When the residual is smaller and projected coefficients are larger, the
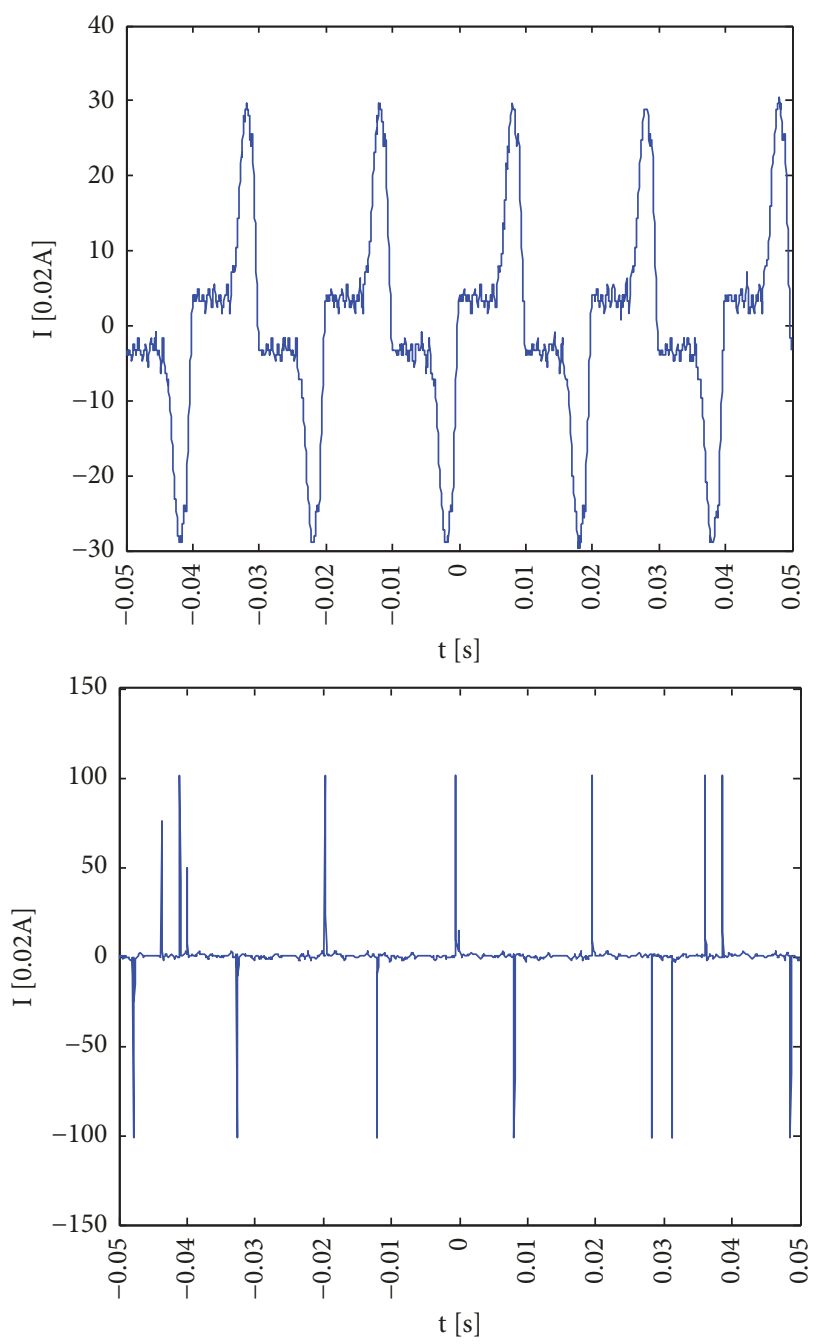

FIGURE 7: Current waveform of switching power load: (a) normal work; (b) arc fault.

corresponding modality is the detection result. The projection coefficients of the horizontal axis 19-36 are larger than those of the horizontal axis 1-18. In Figure 8(b), the residual of the second modality is smaller, so that it is judged to be arc fault and the result is correct. The detection result is shown in Figures 9, 10, 11, and 12, when the single dictionary is $\boldsymbol{D}_{2}, \boldsymbol{D}_{3}, \boldsymbol{D}_{4}$, and $\boldsymbol{D}_{5}$. All the test results of the five sets are arc fault, so the result of final judgment is arc fault, which is consistent with the known result, and the result is correct.

Seventy-two sets of normal work and arc fault data (including resistive load, resistive and inductive load, DC motor load, series motor load, eddy current load, and switching power load) are selected as the test sample. Arc fault is detected by single-dictionary and multidictionary learning, respectively. When single-dictionary learning method is used, seventy sets of results are correct and two sets of results are error. When multidictionary learning method is used, seventy-two sets of results are all correct. Therefore, the arc fault detection based on multidictionary learning method can improve the accuracy. 


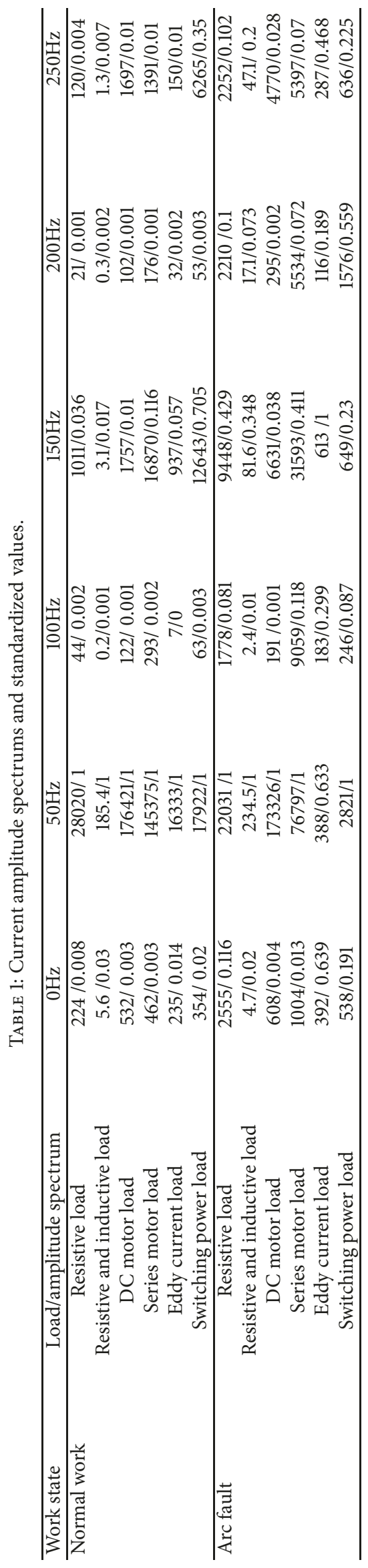



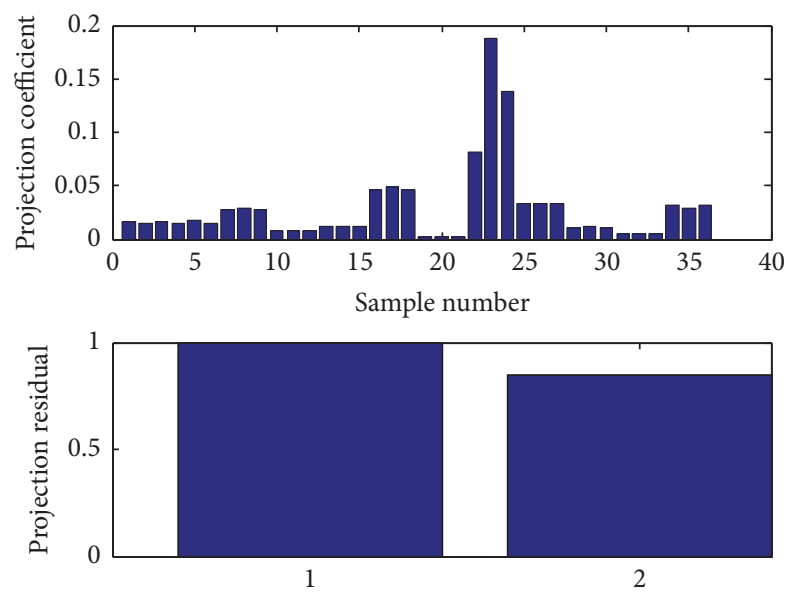

Test result category

Figure 8: Test of $D_{1}$ learning: (a) projection coefficient; (b) residual classification.
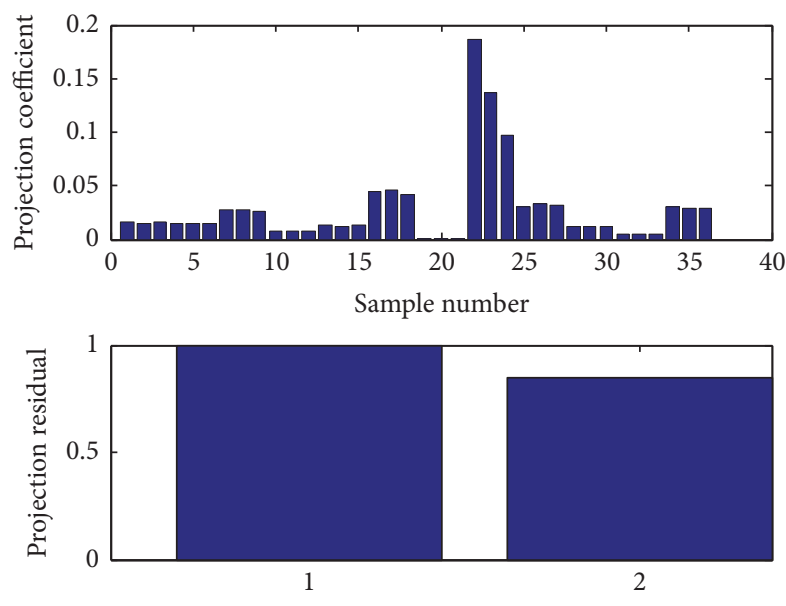

Test result category

FIgURE 9: Test of $D_{2}$ learning: (a) projection coefficient; (b) residual classification.
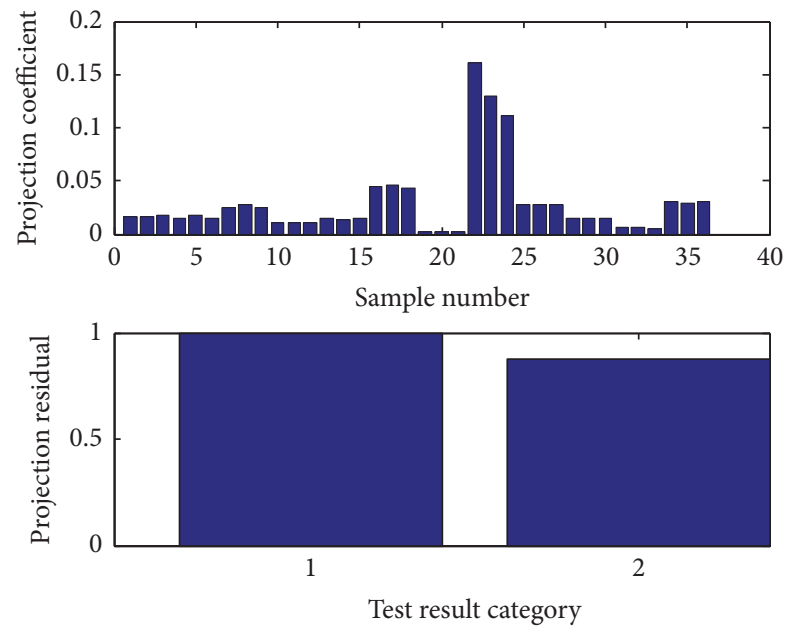

FIGURE 10: Test of $D_{3}$ learning: (a) projection coefficient; (b) residual classification.
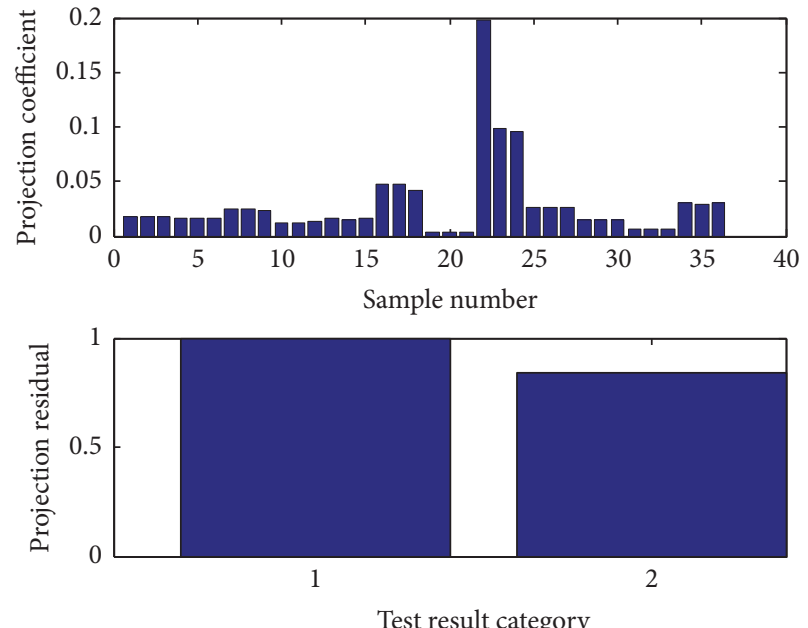

FIGURE 11: Test of $D_{4}$ learning: (a) projection coefficient; (b) residual classification.
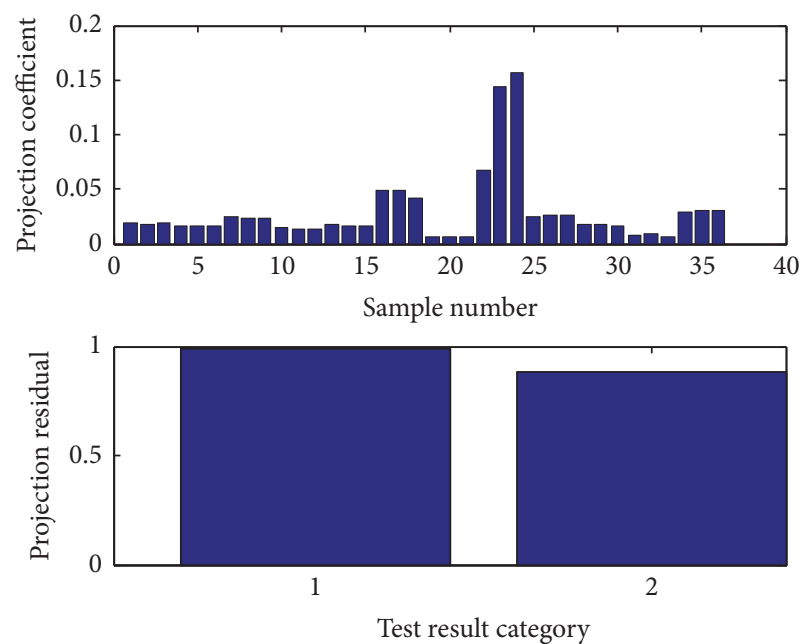

FIGURE 12: Test of $D_{5}$ learning: (a) projection coefficient; (b) residual classification.

Neural network algorithm is the most common method in arc fault detection. Probabilistic neural network (PNN) is a parallel algorithm based on Bayes minimum risk criterion. The proposed algorithm is compared with the PNN by using 72 groups of data. The accuracy of the algorithm is $100 \%$, and that of PNN algorithm is $95.83 \%$ [25]. The accuracy of proposed algorithm is higher than that of PNN algorithm.

\section{Conclusion}

In this paper, we have proposed a new model, which was tailored to detect the arc fault. The proposed model uses the multidictionary learning of sparse representation algorithm. Compared with the algorithm such as neural networks, sparse representation algorithm does not need a lot of repeated trainings. The test data are classified by sparse solution, which can improve the real-time performance and application of 
arc fault detection. Compared with the single-dictionary learning of sparse representation algorithm, multidictionary learning can improve the accuracy of arc fault detection by increasing training samples.

\section{Appendix}

See Table 1.

\section{Data Availability}

The simulation data used to support the findings of this study are available from the corresponding author upon request.

\section{Conflicts of Interest}

The authors declare that there are no conflicts of interest regarding the publication of this paper.

\section{Acknowledgments}

This work was supported by the National Natural Science Foundation of China (61733003 and 61473069) and Department of Education Project of Liaoning Province (L201742).

\section{References}

[1] P. Sun, Z. C. Zheng, and R. G. Yan, "Detection method of arc fault in series with wavelet entropy," Proceedings of the CSEE, vol. 30, pp. 232-236, 2010.

[2] Q. F. Yu, Research on Electrical Fire Forecast System and Its Application Based on Wavelet Analysis and Data Fusion [PhD Thesis], Yanshan University, 2013.

[3] K. Koziy, B. Gou, and J. Aslakson, "A low-cost power-quality meter with series arc-fault detection capability for smart grid," IEEE Transactions on Power Delivery, vol. 28, no. 3, pp. 15841591, 2013.

[4] L. Kumpulainen, G. A. Hussain, M. Lehtonen, and J. A. Kay, "Preemptive arc fault detection techniques in switchgear and controlgear," IEEE Transactions on Industry Applications, vol. 49, no. 4, pp. 1911-1919, 2013.

[5] R. Grassetti, R. Ottoboni, M. Rossi, and S. Toscani, "Low cost arc fault detection in aerospace applications," IEEE Instrumentation \& Measurement Magazine, vol. 16, no. 5, pp. 37-42, 2013.

[6] S. H. Ma, J. Q. Bao, and Z. Y. Cai, "A novel arc fault identification method based on information dimension and current zero," Proceedings of the CSEE, vol. 36, no. 9, pp. 2572-2579, 2016.

[7] J. K. Zhang, Q. L. Deng, and J. C. Tang, "Characteristic analysis and modeling of series arc fault," Electrical Engineering Technology, vol. 1, pp. 30-32, 2017.

[8] S. Li, "Study of low cost arc fault circuit interrupter based on MCU," International Journal of Control and Automation, vol. 8, no. 10, pp. 25-34, 2015.

[9] A. Yaramasu, Y. Cao, G. Liu, and B. Wu, "Aircraft electric system intermittent arc fault detection and location," IEEE Transactions on Aerospace and Electronic Systems, vol. 51, no. 1, pp. 40-51, 2015.

[10] N. L. Georgijevic, M. V. Jankovic, S. Srdic, and Z. Radakovic, "The detection of series arc fault in photovoltaic systems based on the arc current entropy," IEEE Transactions on Power Electronics, vol. 31, no. 8, pp. 5917-5930, 2016.

[11] G. Artale, A. Cataliotti, V. Cosentino, D. Di Cara, S. Nuccio, and G. Tine, "Arc fault detection method based on CZT lowfrequency harmonic current analysis," IEEE Transactions on Instrumentation and Measurement, vol. 66, no. 5, pp. 888-896, 2017.

[12] B.-X. Wang, B.-J. Zhao, L.-B. Tang, S.-G. Wang, and J.-H. Wu, "Robust visual tracking algorithm based on bidirectional sparse representation," Acta Physica Sinica, vol. 63, no. 23, pp. 2342011-234201-11, 2014.

[13] Q.-S. Lian, B.-S. Shi, and S.-Z. Chen, "Research advances on dictionary learning models, algorithms and applications," Acta Automatica Sinica, vol. 41, no. 2, pp. 240-260, 2015.

[14] H. J. Qi, Y. G. Wang, J. Ding, and H. G. Liu, "SAR target recognition based on multiinformation dictionary learning and sparse representation," Systems Engineering and Electronics, vol. 37, no. 6, pp. 1280-1287, 2015.

[15] P. O. Hoyer, "Non-negative matrix factorization with sparseness constraints," Journal of Machine Learning Research, vol. 5, no. 3, pp. 1457-1469, 2004.

[16] Z. Jiang, Z. Lin, and L. S. Davis, "Label consistent K-SVD: learning a discriminative dictionary for recognition," IEEE Transactions on Pattern Analysis and Machine Intelligence, vol. 35, no. 11, pp. 2651-2664, 2013.

[17] S. B. Chen, L. Zhao, and B. Luo, "Kernel fisher discrimination dictionary learning for sparse representation classificatin," Journal of Optoelectronics Laser, vol. 25, no. 10, pp. 89-93, 2014.

[18] S. Bahrampour, N. M. Nasrabadi, A. Ray, and W. K. Jenkins, "Multimodal task-driven dictionary learning for image classification," IEEE Transactions on Image Processing, vol. 25, no. 1, pp. 24-38, 2016.

[19] G. Xia, H. Sun, X. Niu, G. Zhang, and L. Feng, "Keyframe Extraction for Human Motion Capture Data Based on Joint Kernel Sparse Representation," IEEE Transactions on Industrial Electronics, vol. 64, no. 2, pp. 1589-1599, 2017.

[20] Z.-F. Cheng, Y.-B. Zhao, H. Li, and P.-L. Shui, "Sparse representation framework for MIMO radar transmit beampattern matching design," IEEE Transactions on Aerospace and Electronic Systems, vol. 53, no. 1, pp. 520-529, 2017.

[21] J. Wright, A. Y. Yang, A. Ganesh, S. S. Sastry, and Y. Ma, "Robust face recognition via sparse representation," IEEE Transactions on Pattern Analysis and Machine Intelligence, vol. 31, no. 2, pp. 210-227, 2009.

[22] E. Michael, Sparse and Redundant Representations: From Theory to Applications in Signal and Image Processing, Springer, 2010.

[23] N. Qu, J. H. Wang, and L. Jinhai, "An arc fault detection method based on current amplitude spectrum and sparse representation," IEEE Transactions on Instrumentation and Measurement, 2018.

[24] N. Qu, J. Wang, J. Liu, and Z. Li, "Composite fire detection system using sparse representation method," Mathematical Problems in Engineering, vol. 2017, Article ID 6784764, 8 pages, 2017.

[25] F. C. Wu, N. Qu, and X. H. Ren, "Detection of series arc fault based on probabilistic neural network," Application of Electronic Technology, vol. 42, 2018. 


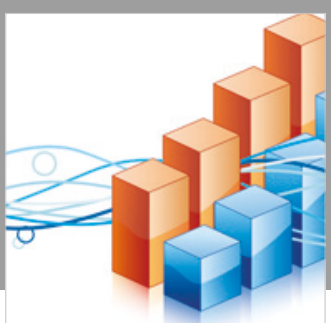

Advances in

Operations Research

\section{-n-m}
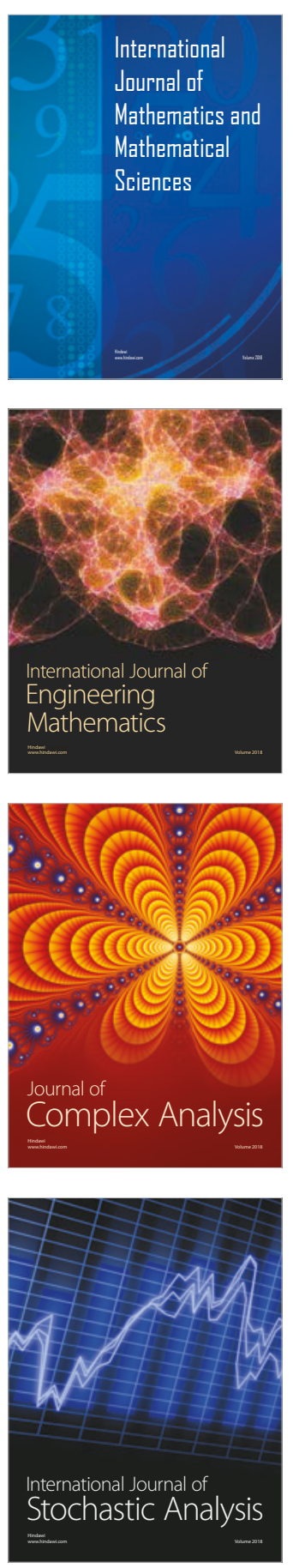
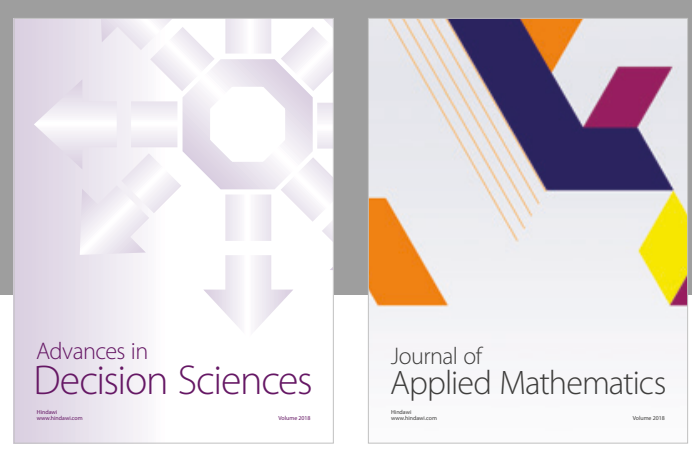

Journal of

Applied Mathematics
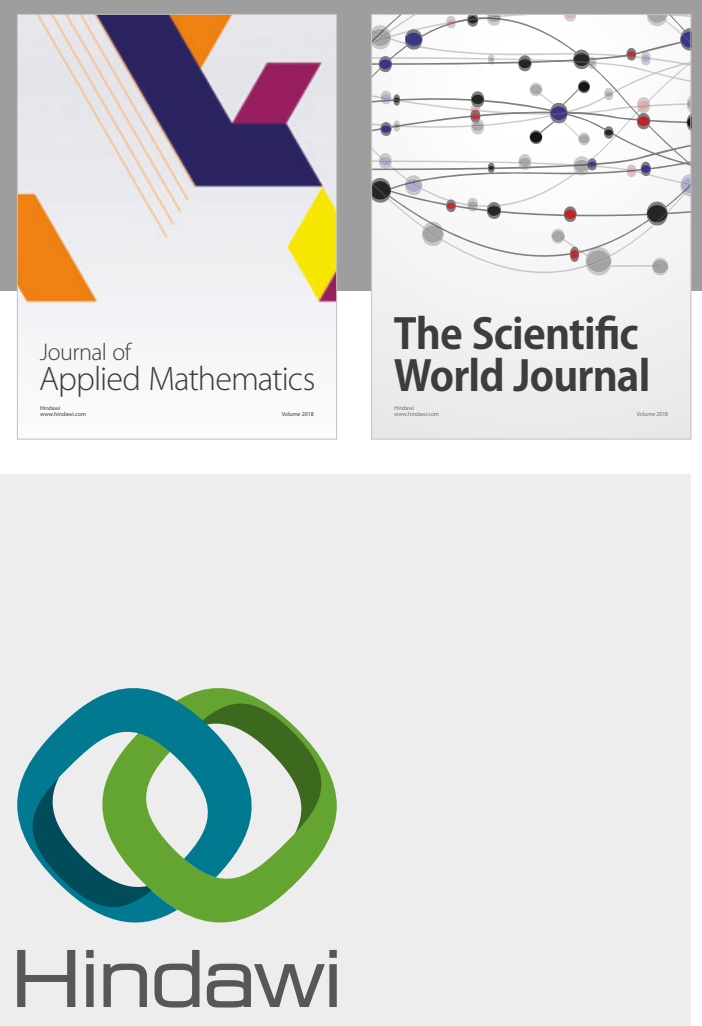

Submit your manuscripts at

www.hindawi.com

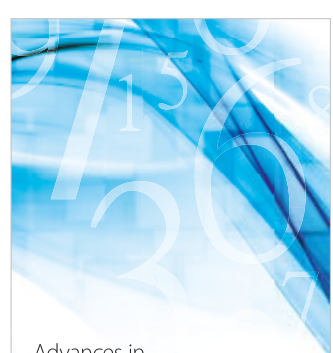

Advances in
Numerical Analysis
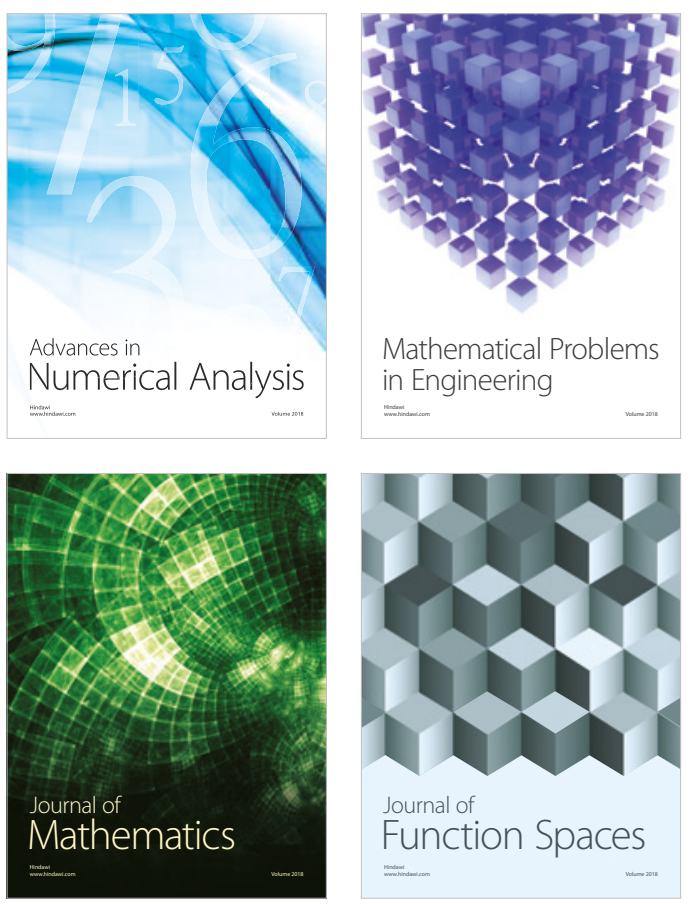

Mathematical Problems in Engineering

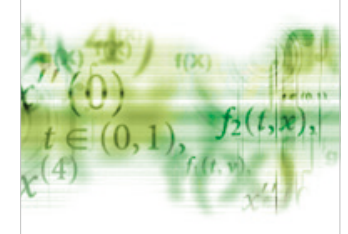

International Journal of

Differential Equations

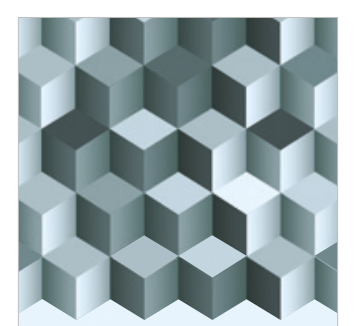

Journal of

Function Spaces

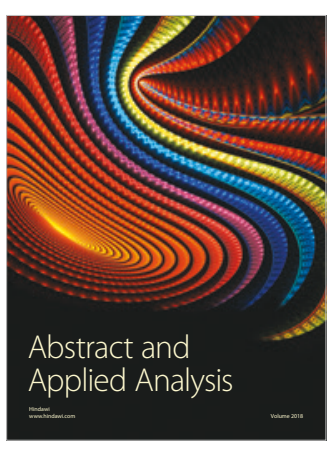

The Scientific

World Journal

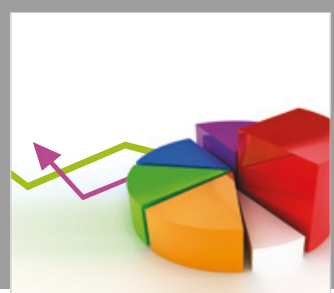

Journal of

Probability and Statistics
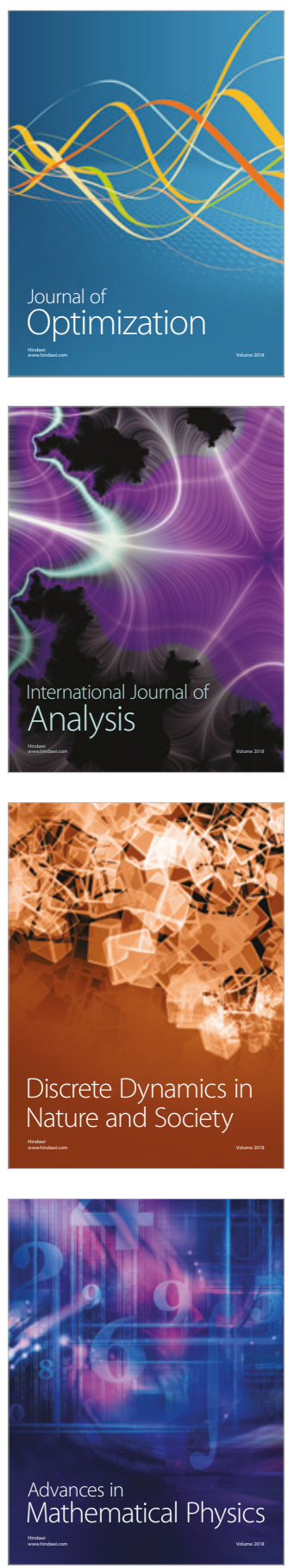